\title{
Trabajo cooperativo basado en SCRUM para desarrollar proyectos distribuidos de visión y robótica
}

\author{
Antonio-José Sánchez-Salmerón ${ }^{a}$, Eugenio Ivorra ${ }^{b}$, Angel Valera ${ }^{c}$ y Carlos Ricolfe- \\ Viala $^{d}$ \\ ${ }^{a}$ Universitat Politècnica de València, e-mail: asanchez@isa.upv.es, ${ }^{\text {b e-mail: euivmar@upvnet.upv.es }}$ \\ c e-mail: giuprog@isa.upv.es y d e-mail: cricolfe@isa.upv.es.
}

\begin{abstract}
Currently, Spanish Universities are introducing masters degree of the Bologna Process approved in 2010. In this current context of transition, professors have to start new subjects of master and think about include new methodologies that improves cross curricular competences that help schoolleavers in their future jobs. For example, learn teamwork skills in order to be an effective group member.

The objective of this study is to explore the Scrum methodology's potential to be incorporated as a didactic strategy based on the development of cooperative computer vision and robotic projects and besides to analyse its possible application to antoher contexts that requires team work and distributed work.

The conclusion is that this methodology is difficult to be incorporated in subjects with more than 50 students and with few lab hours. However, it could be interesting for subjects with lower students and with more lab hours that lets to develop a complete project.
\end{abstract}

Keywords: cross curricular competences; team work;collaborative learning; Scrum

\begin{abstract}
Resumen
Actualmente, las Universidades Españolas están inmersas en la puesta en marcha de los Másteres del Plan de Bolonia aprobado en 2010. En este contexto de cambio, los profesores deben poner en marcha nuevas asignaturas de Master y se deben plantear aplicar nuevas metodologías que favorezcan el desarrollo de algunas competencias transversales importantes para el posterior desempeño de los egresados en sus futuros puestos de
\end{abstract}


Trabajo cooperativo basado en SCRUM para desarrollar proyectos distribuidos de visión y robótica

trabajo, como por ejemplo, aprender a trabajar en cooperación dentro de un equipo. El objetivo de este artículo es explorar el potencial de la metodología Scrum para ser incorporada como una estrategia didáctica de aprendizaje basado en el desarrollo de proyectos cooperativos de visión y robótica y también analizar su posible aplicación a otras situaciones de necesidad de interacción grupal y contexto distribuido.

Se concluye que esta metodología es de difícil aplicación en asignaturas con más de 50 alumnos y con pocos créditos de prácticas. Sin embargo, su implantación puede ser interesante para asignaturas con menos alumnos y con muchos créditos de prácticas que permitán desarrollar un proyecto completo en el laboratorio.

Palabras clave: competencias transversales, trabajo en equipo, aprendizaje colaborativo, Scrum

\section{Introducción}

Actualmente, las Universidades Españolas están inmersas en la puesta en marcha de los Másteres del Plan de Bolonia aprobado en 2010. En este contexto de cambio extructural, los profesores deben poner en marcha nuevas asignaturas de Master y se deben plantear la aplicación de nuevas metodologías que favorezcan el desarrollo de algunas competencias transversales importantes para el posterior desempeño de los egresados en sus futuros puestos de trabajo, como por ejemplo, aprender a trabajar en cooperación dentro de un equipo.

Scrum es una metodología de trabajo para la gestión ágil de proyectos de creciente interés en distintos campos de aplicación (Schwaber, 2010). Este tipo de metodologías resulta especialmente útil, en áreas multidisciplinares como son la robótica y la visión artificial, ya que en estas áreas de desarrollo se suele trabajar en equipo.

Scrum dispone de dos aspectos fundamentales a diferenciar, los actores y las acciones.

Los actores son el Product Owner, el Scrum Master, el Scrum Team y los Usuarios o Clientes. El Product Owner conoce y marca las prioridades del proyecto o producto. El Scrum Master es la persona que asegura el seguimiento de la metodología guiando las reuniones y ayudando al equipo ante cualquier problema que pueda aparecer. El Scrum Team son las personas responsables de implementar la funcionalidad o funcionalidades elegidas por el Product Owner. Los Usuarios o Clientes, son los beneficiarios finales del producto, y son quienes viendo los progresos, pueden aportar ideas, sugerencias o necesidades. 


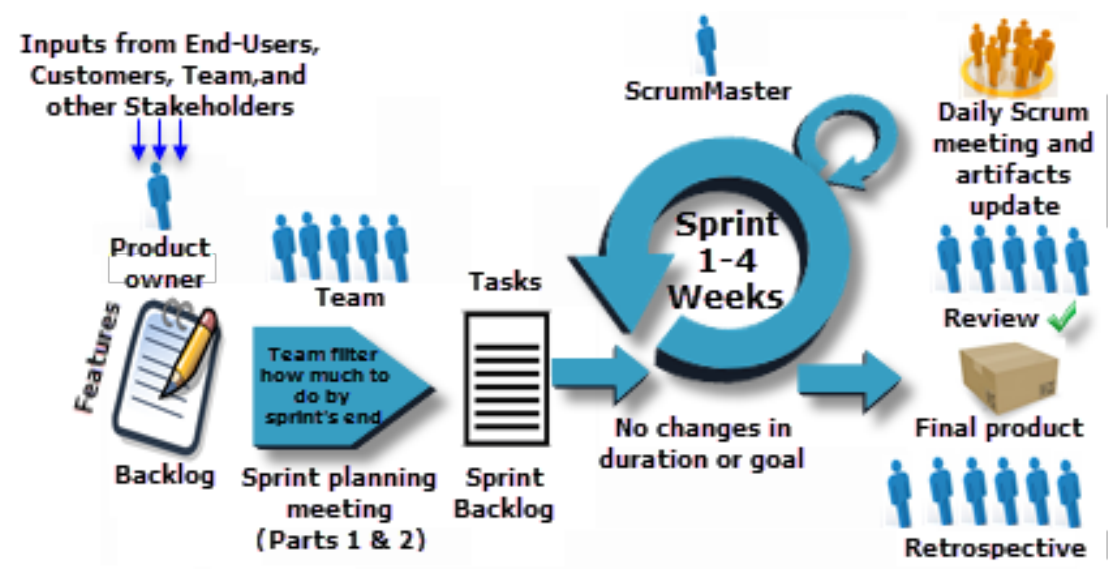

Fig. 1 Metodología Scrum.

Las acciones de Scrum forman parte de un ciclo iterativo repetitivo (Fig. 1), por lo que el mecanismo y forma de trabajar que a continuación se indica, tiene como objetivo minimizar el esfuerzo y maximizar el rendimiento en el desarrollo. Las acciones fundamentales de Scrum son Product Backlog, Sprint Planning Meeting, Sprint Backlog, Daily Scrum Meeting y Sprint Review.

El Product Backlog corresponde a definir todas las tareas, funcionalidades o requerimientos a realizar. El Product Owner es la persona que se encarga de marcar las prioridades, y es al fin y al cabo, la persona que mantiene y actualiza la lista de tareas. El Sprint Planning Meeting es una reunión de todo el equipo que tiene por objetivo, planificar el siguiente Sprint Backlog a partir del Product Backlog. El Sprint Backlog corresponde con una o más tareas que provienen del Product Backlog. Es decir, del Product Backlog se saca una o más tareas que van a formar parte del Sprint Backlog. Las tareas del Sprint Backlog se deben acometer en unas 2 semanas ó 4 semanas. Una norma fundamental es que cuando un Sprint Backlog se inicia, éste no puede ser alterado o modificado. El Daily Scrum Meeting es una tarea que se realiza todos los días del desarrollo de un Sprint Backlog con el equipo de trabajo. Se trata de una reunión operativa, informal y ágil, de un máximo de 30 minutos, en la que se le hace 3 preguntas a cada integrante del equipo. ¿Qué tareas ha realizado desde la última reunión? ¿Qués vas a hacer hoy? ¿Qué problemas puedes tener?

En el Sprint Review se revisa en unas 2 horas como máximo el Sprint finalizado. Al llegar a este punto, debemos tener "algo" que el Cliente o el Usuario pueda ver y tocar. En esta reunión, suelen asistir el Product Owner, el Scrum Master, el Scrum Team y personas que podrían estar involucradas en el proyecto. Al finalizar un Sprint Backlog y el Sprint Review, se inicia el Sprint Retrospective. El Product Owner revisará con el equipo los objetivos marcados inicialmente en el Sprint Backlog concluido, se aplicarán los cambios y ajustes si son necesarios, y se marcarán los aspectos positivos (para repetirlos) y los aspectos negativos (para evitar que se repitan) del Sprint. 
Trabajo cooperativo basado en SCRUM para desarrollar proyectos distribuidos de visión y robótica

\section{Objetivos}

El objetivo principal de este artículo es explorar el potencial de la metodología Scrum para ser incorporado como una estrategia didáctica de aprendizaje basado en el desarrollo de proyectos cooperativos en las áreas de visión y robótica.

\section{Desarrollo de la innovación}

Los autores de este trabajo han aplicado la metodología SCRUM para el desarrollo de los proyectos prácticos durante seis años en dos International Intensive Programs (IPs) titulados "Design of safe and reliable technical systems" (DeSeRTS) durante los cursos 2006/2007, 2007/2008, 2008/2009 y "Safe and secure robotics based on open source SW" (SaSeRoS), durante los cursos 2010/2011, 2011/2012 y 2012/2013. En estos IPs participaron unos 10 profesores y unos 60 alumnos de cinco nacionalidades, alemanes de Fachhochschule Bonn-Rhein-Sieg, holandeses de Hogeschool van Amsterdam, finlandeses de EVTEK Institute of Technology, noruegos de Sor Trondelag University College y españoles de la Universitat Politècnica de València. Estos proyectos se desarrollaron en equipo y consistieron en implementar un robot móvil fiable, basado en las piezas de LEGO Mindstrom (Fig. 2).

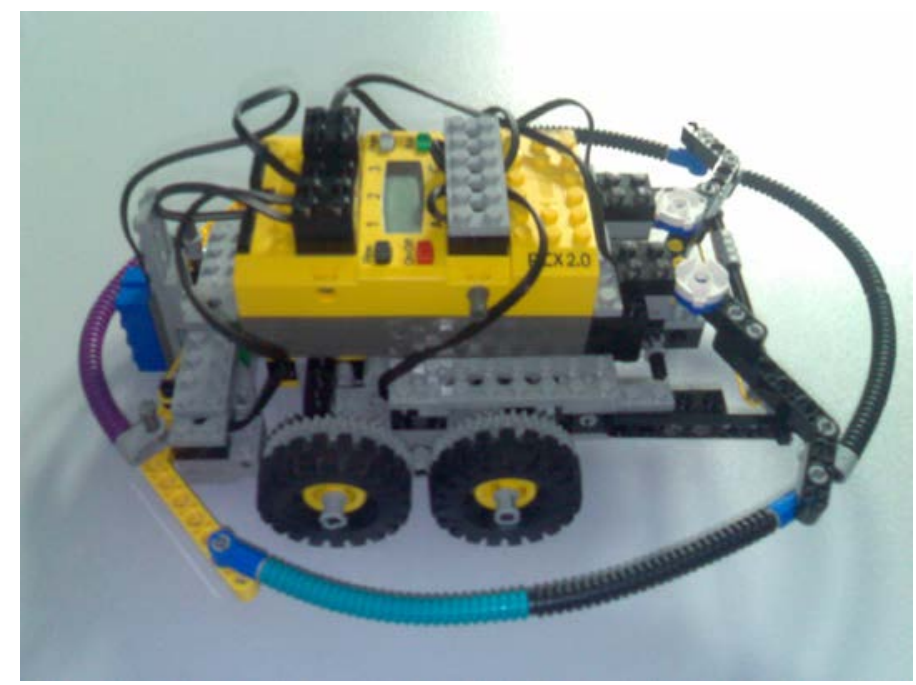

Fig. 2 Ejemplo de robot móvil construido por un equipo de trabajo.

Los equipos de trabajo estaban compuestos por unos 6 estudiantes de distintas nacionalidades que tenían una formación diversa (algunos de ellos eran mecánicos, otros 
electrónicos y otros informáticos). Es decir, los equipos eran multinacionales y multidisciplinares. Cada equipo de trabajo fue dirigido por un profesor.

Tras el éxito de la aplicación de esta metodología en los IPs, los autores han aplicado esta metodología en la asignatura de "Visión Artificial" de cuarto del "Grado en Ingeniería Electrónica Industrial y Automática" de la Escuela Técnica Superior de Ingeniería del Diseño (ETSID) de la Universitat Politècnica de València (UPV) durante los cursos 2012/2013 y 2013/2014. "Visión Artificial" es una asignatura optativa 3 ECTS de teoría de aula, 1 ECTS de práctica de aula y 2 ECTS de práctica de laboratorio en la que se suelen matricular más de 40 alumnos y se dispone de dos grupos de prácticas. Durante estos dos cursos, se crearon equipos de 4 o 5 alumnos que durante los ECTS de prácticas de aula aprendían a desarrollar un proyecto cooperativo de visión de forma guiada por un profesor y durante los ECTS de prácticas de laboratorio desarrollaban de forma más autónoma un proyecto cooperativo por equipos. En este caso todos los equipos fueron dirigidos por un solo profesor.

Por otra parte, durante el último curso 2014/2015 en esta misma asignatura de "Visión Artificial" se aplicó la metodología típica de prácticas por grupos de dos alumnos, para los ECTS de práctica de laboratorio. En este caso se desarrollaron varios mini-proyectos de forma guiada por el profesor, en vez de un solo proyecto cooperativo de forma más autónoma.

\section{Resultados}

Al igual que confirman otras experiencias de aplicación de Scrum (Yazyi, 2011), la satisfacción por parte del alumnado y profesorado durante el desarrollo de los IPs fue muy positiva. Sin embargo, la satisfacción de los profesores de prácticas de la asignatura "Visión Artificial" fué más positiva en el curso 2014/2015 al utilizar la metodología tradicional que al utilizar la metodología Scrum durante los dos cursos anteriores.

El principal problema encontrado al aplicar Scrum en la asignatura de "Visión Artificial" fue que algunos equipos de trabajo necesitaban mucha ayuda por parte del profesor de prácticas para ser capaces de resolver los problemas encontrados durante el desarrollo del proyecto. Por lo que un solo profesor no pudo atender debidamente las demandas de tantos equipos de trabajo.

Por otra parte también se detectó que algunos alumnos no se integraron en las labores del equipo y quedaron al margen. Este último problema ocurrió sobre todo porque algunas herramientas de desarrollo no estaban preparadas para permitir trabajar en equipo. Por lo tanto, de cara a poder implantar esta metodología de trabajo, se deberían utilizar herramientas de desarrollo que faciliten trabajar en equipo. Estas herramientas consisten en 
gestores de proyectos y programación colaborativa tales como ICESCRUM que es un programa de gestor de proyectos basados en Scrum de código libre y ETHERPAD que es un editor online cooperativo que permite programar en diferentes lenguajes de programación y que al igual que ICESCRUM, es de código libre.

El departamento de Ingenieria de Sistemas y Automática pasa todos los años una encuesta para medir el grado de satisfacción de los alumnos en las prácticas de todas las asignaturas que imparte. Los resultados de estas encuestas de satisfacción del alumnado en el curso 2014/2015 han mejorado un punto con respecto a los años anteriores, por lo que en principio parece que los alumnos también prefieren la metodología tradicional.

\section{Conclusiones}

De la experiencia trianual de aplicación de Scrum en "Visión Artificial", se concluyó que la metodología SCRUM no es la más adecuada para asignaturas donde los equipos no tienen la preparación necesaria para ser lo suficientemente autónomos para que un solo profesor de prácticas pueda guiar a 4 o 5 equipos de manera satisfactoria. Además en esta asignatura solo se dispone de 2 ECTS de prácticas de laboratorio por lo que el proyecto a desarrollar debe ser muy corto.

Sin embargo, su implantación puede ser muy interesante para nuevas asignaturas de Master con menos alumnos y con muchos créditos de prácticas que permitirán desarrollar un proyecto completo en el laboratorio, siempre y cuando se dispongan de herramientas de desarrollo cooperativas. En este sentido se propone utilizar herramientas que facilitan el desarrollo cooperativo de proyectos de visión y robótica, por lo que se facilita la aplicación de Scrum como metodología de trabajo cooperativo.

\section{Referencias}

[1] ETHERPAD, The Etherpad Foundation., http://etherpad.org (Consultado el 27-05-2015)

[2] ICESCRUM, Kagilum SAS, wWw.icescrum.org (Consultado el 27-05-2015)

[3] Schwaber, K. Advanced Development Methods. SCRUM Development Process Retrieved July 01, 2010.

[4] Yazyi, S. A. (2011). Una experiencia práctica de Scrum a través del aprendizaje basado en proyectos mediado por TIC en un equipo distribuido. Tesis de Máster. Universidad de Salamanca. 